\title{
Evaluation of Gastro-Esophageal Reflux Disease after Sleeve Gastrectomy in Morbid Obese Patients \\ ${ }^{1}$ Mohammed Elsayed Radwan, ${ }^{1}$ Mohammed Arafat Abdel-Maksoud, ${ }^{2}$ Ashraf Abdo-Almonem Sayed Ahmad Abotaleb, ${ }^{1}$ Sobhy Rezk Ahmed Teama \\ ${ }^{1}$ Department of General Surgery, ${ }^{2}$ Department of Clinical Pathology, Faculty of Medicine, Al-Azhar University \\ Corresponding author: Sobhy Rezk Ahmed Teama, Mobile: 01061757521 Email: sobhyteama2007@ gmail.com
}

\begin{abstract}
Background: obesity is one of the major problems in the world and is associated with several comorbidities and disabling diseases e.g. cardiovascular disease, metabolic syndrome, type 2 diabetes mellitus (T2DM), infertility, certain tumor types, and GERD. Management consisted of conservative and surgical measures, conservative measures such as diet modifications physical exercise and pharmacological therapy. There was considerable evidence in the literature on the long-term positive impact of bariatric surgery as a primary therapy for the treatment of obesity and its co-morbidities. Objective: this study aimed to conclude that if gastroesophageal reflux disease develops after sleeve gastrectomy in morbidly obese patients or not. Patients and Methods: this prospective non-comparative study was conducted in Surgery Department, Al-Azhar University Hospitals in the period between October 2016 and October 2018. Study included 30 cases of morbidly obese patients (with BMI $\geq 40$ $\mathrm{Kg} / \mathrm{m}^{2}$ or $\geq 35 \mathrm{Kg} / \mathrm{m}^{2}$ associated with comorbidities) and they were submitted for laparoscopic sleeve gastrectomy and all of them had failed in trials of conservative management including dietary control and they are bulky eater but non-sweet eater. Results: the prevalence of gastroesophageal reflux disease (GERD) was markedly increased in morbid obese patients compared to the incidence in normal individuals, with a percentage of up to $70 \%$ of patients undergoing bariatric surgery. SG is associated with an increase in GERD prevalence. The measured increase in GERD prevalence ranged from $2.1 \%$ to $34.9 \%$ in the analyzed literature. There was marked heterogeneity between the studies in regard to a number of factors including preoperative BMI, method of evaluating GERD, exclusion criteria, length of follow-up and operative technique. Conclusion: only in a very special group of patients with BMI between 30 and 35 and comorbidities, SG plus other antireflux procedure or hiatal hernia repair is accepted.
\end{abstract}

Keywords: central nervous system, gastro-esophageal reflux disease, laparoscopic sleeve gastrectomy

\section{INTRODUCTION}

Obesity is a chronic medical condition in which excess body fat has accumulated to extend that it may has an adverse effect on health, leading to reduction of life expectancy or increasing health hazards. Obesity and its related co-morbidities are increasing to epidemic proportions at an alarming rate worldwide. It is estimated that more than 300 million adults worldwide are obese (Body Mass Index [BMI] $>30 \mathrm{~kg} / \mathrm{m}^{2}$ ) and $20 \%$ of them are morbidly obese $\left(\mathrm{BMI}>35 \mathrm{~kg} / \mathrm{m}^{2}\right)^{(\mathbf{1})}$. In Taiwan, the incidence of morbidly obese patients (BMI $>35 \mathrm{~kg} / \mathrm{m}^{2}$ ) had doubled the incidence in the past decade and consisted of $1.5 \%$ of the population in a recent survey ${ }^{(2)}$.

Obesity prevalence is increasing rapidly worldwide, in both developed and developing countries, a number of serious comorbidities are associated with morbid obesity, apnea, gastroesophageal reflux disease (GERD), nonalcoholic fatty liver disease and increase incidence of different tumors ${ }^{(3)}$. An additional common weight-related comorbidity is gastroesophageal reflux disease (GERD), with an increase in both reflux symptoms and esophageal adenocarcinoma associated with a body mass index above $30^{(4)}$. The Montreal Classification defines GERD as a condition that develops when reflux of stomach contents in the esophagus causes troublesome symptoms or complications. Symptoms associated with GERD include heartburn, dysphagia, regurgitation laryngitis, and chronic cough. Prolonged acid exposure within the esophagus can lead to histopathologic and structural changes as peptic stricture and Barrett's esophagus ${ }^{(5)}$. Bariatric surgery is the most effective treatment for morbid obesity and the long-term results regarding weight loss and improvement of obesity related co-morbidities (6). Sleeve gastrectomy (SG) was seen just as a part of the biliopancreatic diversion with 
duodenal switch (BPD-DS). Soon, SG was being considered as an isolated procedure to treat obesity due to the nice association of physical and neuroendocrine modifications. Because SG may produce excellent results achieving very high quality of life with smaller changes in the general structure of the gastrointestinal tract, it has become very popular worldwide ${ }^{(7)}$. The prevalence of gastroesophageal reflux disease GERD is markedly increased in morbid obese patients compared to the incidence in normal individuals, with a percentage of up to $70 \%$ of patients undergoing bariatric surgery ${ }^{(8)}$. SG is associated with an increase in GERD prevalence. The measured increase in GERD prevalence ranged from $2.1 \%$ to $34.9 \%$ in the analyzed literature. There was marked heterogeneity between the studies in regard to a number of factors including preoperative BMI, method of evaluating GERD, exclusion criteria, length of follow-up and operative technique ${ }^{(\boldsymbol{9})}$. In another analyzed literature, GERD prevalence decreases between $2.8 \%$ and $20 \%$ after SG. Like the papers demonstrating increased GERD prevalence, there was a significant amount of heterogeneity between the studies. The study with the largest decrease in GERD prevalence was detected by Weiner et al. ${ }^{(\mathbf{1 0})}$ in their prospective single center study. They demonstrated a 20\% decrease in GERD prevalence ${ }^{(9)}$. The effect of SG on GERD remains controversial. There remains marked heterogeneity among the studies assessing GERD following SG. Assessment using a common objective standard for the evaluation of GERD, such as 24-hour $\mathrm{pH}$ monitoring, is lacking. Differences in surgical technique among studies may also contribute to difficulty comparing GERD following SG in the literature (9).

\section{AIM of the WORK}

This work aimed to conclude that if gastroesophageal reflux disease develops after sleeve gastrectomy in morbidly obese patients or not.

\section{PATIENTS and METHODS}

\section{Patient selection and preoperative preparation}

This prospective non-comparative study was conducted in Surgery Department, Al-Azhar University Hospitals in the period between October 2016 and October 2018. Study included 30 cases of morbidly obese patients (with BMI $\geq 40 \mathrm{Kg} / \mathrm{m}^{2}$ or $\geq 35 \mathrm{Kg} / \mathrm{m}^{2}$ associated with comorbidities) and they were submitted for laparoscopic sleeve gastrectomy and all of them had failed in trials of conservative management including dietary control and they are bulky eater but non-sweet eater.

Different options for management of morbid obesity were discussed in details with the patients with emphasis on the benefits and more importantly the potential complications and side effects of the LSG. The study was approved by the Ethics Board of Al-Azhar University.

Inclusion criteria: all cases were chosen according to the following criteria: BMI more than 40 or more than 35 with obesity associated co-morbidity, age between 18 - 60 years, no endocrinal causes for obesity, psychologically stable patients, patients without GERD or hiatus hernia, motivation and acceptance of surgical risks. Cooperative with follow-up period

Exclusion Criteria: exclusion criteria included all patients with psychiatric impairment or those with BMI less than 40 $\mathrm{Kg} / \mathrm{m}^{2}$ or less than $35 \mathrm{Kg} / \mathrm{m}^{2}$ and not associated with other comorbidities or those who had previous abdominal surgery or other contraindications for laparoscopic surgery or abnormally sweet (high caloric fluid) eater, younger than 18 years or older than 60 years, pregnant or breast feeding, patients suffering from any severe psychiatric illness, patients with GERD or hiatus hernia, patient not accepted post-operative follow up.All patients were subjected to full clinical preoperative evaluation (personal and medical) as well as full laboratory investigations (as full blood count, liver function tests, kidney function tests, liver enzymes, thyroid function; $\mathrm{T}_{3}, \mathrm{~T}_{4}, \mathrm{TSH}$ and lipid profile).

\section{Patient's workup}

Clinical evaluation aimed at assessment of degree of obesity, preoperative evaluation and detection of different complications of morbid obesity like hypertension, DM, sleep apnea, skeletal problems, infertility, hernias, history of psychotherapy, history of reflux symptoms (acidic taste in the mouth, regurgitation, and heartburn). 


\section{Investigation}

Laboratory investigations: CBC, FBS, renal functions, liver functions, coagulation profile, lipid profile. Hormonal assay: to detect any endocrinal causes of obesity as hypothyroidism. Pulmonary evaluation included: chest X-ray and pulmonary function tests, radiological investigation abdominal U /S to evaluate the patients for any abdominal abnormality as gall bladder stones or biliary disorders and to exclude them from the study. Cardiac assessment: ECG and echocardiography if needed, Duplex U/S on both lower limbs Upper GIT series: esophagogastroduodenoscopy. The goals of the preoperative assessment for bariatric surgery are to assess indications and contraindications to bariatric surgery and to treat or optimize medical comorbidities before the surgical treatment. Moreover, another aim is to educate the patients and their families about options and risks of the procedures and to set realistic expectations.Unless there was a clinical need for early admission, all the patients are admitted on the day of the surgery. In uneventful cases we aimed for discharge one day postoperative. Patient Preparation: patient counselling and consent, prophylactic clexane (enoxaparin) and compression boots for DVT prophylaxis, prophylactic antibiotics e.g. $3^{\text {rd }}$ generation cephalosporins and Foley catheter for urine output monitoring.

\section{Operative steps}

In this study, 30 patients were included and operated by 2 surgeons with the same technique.Patient was placed on the operating table in the supine position with the operating surgeon between the legs of the patient. The preferred position for operating was using the full incline of the table in the anti-trendlenberg position. However, during the port placement, the patient was placed in the supine position.

A pneumoperitoneum is then established to $15-\mathrm{mmHg}$ pressure carbon dioxide using Veress needle inserted $3 \mathrm{~cm}$ below left subcostal border in midclavicular line (Palmer's point). Optical entry is the preferred method of entry to the abdominal cavity with $12-\mathrm{mm}$ trocar loaded with the 10$\mathrm{mm}$ 0-degree laparoscope under laparoscopic observation. This laparoscope is then changed to a 30 or 45-degree scope.The classic configuration is a $10 \mathrm{~mm}$ port in the supraumbilical position for a $30^{\circ}$ scope, two additional $12 \mathrm{~mm}$ ports at each flank to serve as the working channel for the leading surgeon, 10 $\mathrm{mm}$ port located at the epigastrium for retraction of the liver, and a final more lateral 5 $\mathrm{mm}$ port at the left flank to perform traction of the omentum and the stomach. A nasogastric tube is inserted at the beginning to decompress the stomach. A window is dissected at the junction of the greater curvature and the greater omentum, around $10 \mathrm{~cm}$ from the pylorus. Division of the gastroepiploic, short gastric and posterior fundic vessels is done starting at $4 \mathrm{~cm}$ proximal to the pyloric ring all the way till the angle of His using the ultrasonic sealing device. Once the dissection part is over, a $36 \mathrm{Fr}$ bougie is introduced orally by the anesthesiologist through the oesophagus and inside the stomach. The surgeon then guides it along the lesser curvature and into the pyloric channel and duodenal bulb.

Gastric transection begins $4 \mathrm{~cm}$ proximal to the pylorus. A $60-\mathrm{mm}$, green or gold cartilage is placed across the antrum through the right midepigastric port and fired.

The second stapler is placed approximately 1 to $2 \mathrm{~cm}$ from the border of the lesser curvature in the direction of the gastroesophageal junction. The bougie must be held in position during this part of the procedure until completion of the stomach transection to avoid stapling across a displaced bougie.

Sequential firings of the stapler were done along the border of the bougie on the lesser curvature completes the gastric transection at the left crus. After completing the transection the entire staple line is inspected carefully to make sure that the staples are well formed especially at the antrum where the stomach is thickest. The transected part of the stomach is then removed through one of the 12 $\mathrm{mm}$ port sites.

After the transection has finished the hemostasis is checked then the bougie is removed followed by insertion of nasogastric tube into the stomach through which methylene blue is injected to confirm that no leak. A $22 \mathrm{Fr}$ nelaton catheter is inserted at staple line then we removed all ports and camera at end.

All fascial defects at ports 10 and 12 were closed by vicryl 0 to prevent hernias. Then patient is recovered and transferred to ward or ICU for early post-operative care.

\section{Statistical analysis}


The collected data were revised, coded, tabulated and introduced to a PC using Statistical package for Social Science (IBM Corp. Released 2011. IBM SPSS Statistics for Windows, Version 20.0. Armonk, NY: IBM Corp). Data was presented and suitable analysis was done according to the type of data obtained for each parameter. Description of quantitative variables as mean, SD and range with escription of qualitative variables as number and percentage.

\section{RESULTS}

Table 1: age and sex distribution

\begin{tabular}{|l|c|}
\hline & Value (\%) \\
\hline Female & $21(70 \%)$ \\
\hline Male & $9(30 \%)$ \\
\hline $\begin{array}{l}\text { Age, Mean } \\
\text { (years) SD, Range }\end{array}$ & $40 \pm 2.7(20-60)$ \\
\hline
\end{tabular}

Table 2: demographic data distribution

\begin{tabular}{|l|l|}
\hline & Mean \pm SD (Range) \\
\hline Body weight $(\mathbf{k g})$ & $134.5 \pm 32.7(110-164)$ \\
\hline Height $(\mathbf{m})$ & $1.68 \pm 0.2(1.58-1.8)$. \\
\hline BMI $\left(\mathbf{k g} / \mathbf{m}^{\mathbf{2}}\right)$ & $50.7 \pm 15.4(35-55)$ \\
\hline
\end{tabular}

Table 3: pre-operative co-morbidities

\begin{tabular}{|l|c|c|}
\hline Co-morbidity & Number & Percent \\
\hline Hypertension & 8 & $26.67 \%$ \\
\hline Type II diabetes mellitus & 13 & $43.33 \%$ \\
\hline Hyperlipidemia & 19 & $63.33 \%$ \\
\hline
\end{tabular}

Table 4: BMI reduction on the follow up period

\begin{tabular}{|l|c|}
\hline BMI & Mean \pm SD $\left(\mathbf{k g} / \mathbf{m}^{\mathbf{2}}\right)$ \\
\hline Initial & $50.7 \pm 15.4$ \\
\hline 2 months & $42.6 \pm 10.2$ \\
\hline 4 months & $38.4 \pm 12.3$ \\
\hline 6 months & $35 \pm 14.6$ \\
\hline
\end{tabular}

Table 5: weight reduction on the follow up period

\begin{tabular}{|l|c|}
\hline Weight & Mean \pm SD $(\mathbf{k g})$ \\
\hline Initially & $134.5 \pm 32.7$ \\
\hline 2 months & $115.9 \pm 18.1$ \\
\hline 4 months & $101.1 \pm 17.8$ \\
\hline 6 months & $93.8 \pm 10.2$ \\
\hline
\end{tabular}

Table 6: post-operative resolution of comorbidities

\begin{tabular}{|l|c|c|}
\hline Co-morbidity & $\begin{array}{c}\text { Resolved } \\
(\%)\end{array}$ & Number \\
\hline Hypertension & $37.5(\%)$ & $3 / 8$ \\
\hline Type 2 diabetes mellitus & $53.84(\%)$ & $7 / 13$ \\
\hline Hyperlipidemia & $57.89(\%)$ & $11 / 19$ \\
\hline
\end{tabular}

Table 7: post-operative GERD

\begin{tabular}{|l|c|c|}
\hline Post-operative GERD & N & \% \\
\hline Yes & 8 & $\begin{array}{c}26.67 \\
\%\end{array}$ \\
\hline
\end{tabular}

\begin{tabular}{|l|c|c|}
\hline No & 22 & $\begin{array}{c}73.33 \\
\%\end{array}$ \\
\hline
\end{tabular}

Table 8: GERD symptoms postoperatively

\begin{tabular}{|l|c|c|}
\hline \multicolumn{1}{|c|}{} & N. Total (8) & \% \\
\hline Heartburn & 7 & $87.5 \%$ \\
\hline Regurgitation & 5 & $62.5 \%$ \\
\hline Epigastric or chest pain & 3 & $37.5 \%$ \\
\hline Epigastric fullness & 3 & $37.5 \%$ \\
\hline Dysphagia & 2 & $25.0 \%$ \\
\hline Cough & 2 & $25.0 \%$ \\
\hline
\end{tabular}

Table 9: endoscopic findings 2, 4 and 6 months after sleeve gasterectomy

\begin{tabular}{|l|c|c|c|}
\hline Endoscopy & $\begin{array}{c}\mathbf{2} \\
\text { months }\end{array}$ & $\begin{array}{c}\mathbf{4} \\
\text { months }\end{array}$ & $\begin{array}{c}\mathbf{6} \\
\text { months }\end{array}$ \\
\hline $\begin{array}{l}\text { Dilated cardia } \\
\text { without esophagistis }\end{array}$ & $\begin{array}{c}1 \\
(12.5 \%)\end{array}$ & $2(25 \%)$ & $\begin{array}{c}4(50 \\
\%)\end{array}$ \\
\hline $\begin{array}{l}\text { Dilated cardia with } \\
\text { esophagitis }\end{array}$ & $6(75$ & 5 & 3 \\
& $\%)$ & $(62.5 \%)$ & $(37.5 \%)$ \\
\hline Hiatal hernia & 1 & 1 & 1 \\
$(12.5 \%)$ & $(12.5 \%)$ & $(12.5 \%)$ \\
\hline Total & $\mathbf{8}$ & $\mathbf{8}$ & $\mathbf{8}$ \\
\hline
\end{tabular}

Table 10: treatment of GERD postoperatively

\begin{tabular}{|l|c|c|}
\hline & $\begin{array}{c}\text { N (Total } \\
\text { 8) }\end{array}$ & $\%$ \\
\hline Improvement of symptoms & 3 & $\begin{array}{c}\text { \% } \\
\%\end{array}$ \\
\hline Continious on PPIs & 5 & $\begin{array}{c}62.5 \\
\%\end{array}$ \\
\hline
\end{tabular}

\section{DISCUSSION}

Obesity is one of the most important public health problems in the United States and many other resource-rich countries and transitional economies ${ }^{(\mathbf{1 1})}$.

As the prevalence of obesity increased, so did the prevalence of the comorbidities associated with obesity. For this reason, it is imperative that health care providers identify overweight and obese patients so that treatment can be provided ${ }^{(12)}$.

A number of comorbidities were associated with morbid obesity, including type 2 diabetes mellitus $\left(\mathrm{T}_{2} \mathrm{DM}\right)$, hypertension, osteoarthritis, obstructive sleep apnea, gastroesophageal reflux disease (GERD), nonalcoholic fatty liver disease and increase incidence of different tumors ${ }^{(3)}$.Laparoscopic sleeve gastrectomy (LSG) had shown excellent results in weight loss and resolution of comorbidities. Despite that, the effect of LSG on gastroesophageal reflux disease (GERD) is still a controversial ${ }^{(13)}$.

Evolution of gastroesophageal reflux disease (GERD) after sleeve gastrectomy (SG) is controversial topic. Some authors reported worsening or improvement of preoperative GERD, others report the occurrence of de novo 
GERD between 5 and $69 \%{ }^{(14)}$. This prospective non-comparative clinical study was carried out on 30 patients. They were assessed by multidisciplinary (MDT) team to assess their psychological and nutritional status and other aspects.Among the studied participants, 21 were females $(70 \%)$ and 9 were males $(30 \%)$. Patients' ages ranged from 20 - 60 years old, with a mean age of 44.As regard to GERD, all patients were free from symptoms and upper GI endoscopy was free for all patients too and30 patients were included and operated by 2 surgeons with the same technique.Initial weight was $134.5 \mathrm{~kg}, 2$ months postoperatively it was $115.9 \mathrm{~kg}, 4$ months postoperatively it reaches $101.1 \mathrm{~kg}^{2}$ and on 6 months postoperatively it was decreased to $93.8 \mathrm{~kg}$ finally. Our reported weight loss results are matched with other published data ${ }^{(\mathbf{1 0 , 1 5 , 1 6})}$. Initial BMI was 50.7 $\mathrm{kg} / \mathrm{m}^{2}, 2$ months postoperatively it was 42.6 $\mathrm{kg} / \mathrm{m}^{2}, 4$ months postoperatively it reaches 38.4 $\mathrm{kg} / \mathrm{m}^{2}$ and finaly on 6 months postoperatively it was decreased to $35 \mathrm{~kg} / \mathrm{m}^{2}$. This finding is matched with another studies ${ }^{(\mathbf{1 0}, \mathbf{1 6})}$ with weight BMI reduction of $15.7,14,12.2,12.8\left(\mathrm{~kg} / \mathrm{m}^{2}\right)$ respectively.

In contrast, some reports as Himpens et al. reported a small reduction in BMI on follow up to be $8.7\left(\mathrm{~kg} / \mathrm{m}^{2}\right)^{(17)}$ while, reduction was not reported in study of Santoro ${ }^{(18)}$.Operative time in minutes was $106 \pm 15.8$ $($ Mean \pm SD), with the minimal value of 58 minutes and maximum value was 212 minutes. There was remarkable resolution of comorbidities in most patients, including hypertension, type 2 diabetes mellitus and hyperlipidemia. Gastroesophageal reflux disease (GERD) was diagnosed by patient symptoms and upper GI endoscopy. This was done on preoperative basis.

Postoperatively, symptomatic patients were re-evaluated by GI endoscopy on 2, 4 and 6 month periods. In our study, symptoms of GERD were evaluated via written questionnaire post operatively. This involved Heartburn, regurgitation, epigastric or chest pain, epigastric fullness, dysphagia and cough. After 2 months postoperatively, clinical questionnaire to confirm the appearance of reflux symptoms, mainly heartburn, regurgitation, or vomiting was done.

All patients with GERD symptoms were submitted to upper GIT endoscopy on $2^{\text {nd }}$, $4^{\text {th }}, 6^{\text {th }}$ months postoperatively. Endoscopy was done to evaluate the distal esophageal mucosa and the tubulized stomach to exclude the presence of distal esophagitis, gastritis, ulcer, or stricture of the gastric segment.

GERD occurred in $26.67 \%$ (8/30) of patients. 22 out of the 30 patients were free from any symptoms of GERD $(73.33 \%)$.

Multiple studies reported the incidence of new-onset GERD symptoms, ranging from 0 $\%$ to $34.9 \%{ }^{(\mathbf{1 9 , 2 0})}$. Sleeve gastrectomy was proposed to have an adverse effect on the function of the lower esophageal sphincter owing to gastric resection of the angle of His predisposing to postoperative reflux symptoms. Concerning results of endoscopic evaluation after sleeve gastrectomy, there are few data mentioning endoscopic erosive esophagitis in current available literature ${ }^{(\mathbf{2 1})}$.

Dietel et al. published the experiences reported in the First International Consensus Summit for SG (ICSSG) and postoperative GER resulted in $4.7 \pm 8.9 \%$ (range 0 to $36 \%$ ) after SG ${ }^{(15)}$. More precisely, regarding exclusively reflux symptoms after sleeve gastrectomy, the rate is variable ranging from $2.8 \%$ to $13 \%{ }^{(22,23)}$.

The relationship between GERD and SG was multifactorial. The factors that increase GERD after SG include a reduction of LES pressure (possibly from division of ligaments and blunting of the angle of His), a reduction in gastric compliance and emptying, increased sleeve pressure, and decreased sleeve volume and distensibility. These GERD exacerbating factors can be countered by accelerated gastric emptying and weight loss. Finally, the resolution of GERD in the long term can be accounted for by the increased gastric compliance and emptying and restoration of the angle of His at 3 years after SG.

Reports that considered SG as a refluxogenic procedure as ${ }^{(18)}$ postulated some mechanisms for reflux after GS as concomitant presence of a hiatal hernia, dissection of the phrenoesophageal ligament, intact or incompetent pylorus with a narrow gastric tube, intrathoracic sleeve migration, narrowing at the incisura, or fundus regrowth ("neostomach") $(17,21)$.

Our study searched for de novo GERD in a clinically negative patients prior to the operation. Multiple studies reviewed the prevalence of GERD after SG ${ }^{(22,23)}$.

They found an increased prevalence of GERD symptoms after SG, but others showed 
a reduced prevalence ${ }^{(\mathbf{1 0 , 1 7})}$. From the studies in which an overall reduced prevalence was reported, the investigators had noted that patients with pre-existing GERD had shown improvement but that new cases of GERD had developed after SG ${ }^{(17)}$. Himpens et al. noted that the patients with pre-existing GERD, 75\% had had resolution. However, $21.8 \%$ new cases had developed at 1 year after SG ${ }^{(17)}$. Melissas et al. observed the same trend of a reduction in pre-existing GERD but also 2 new cases ${ }^{(24)}$. However, the studies did not report the statistical significance of the new cases. Study of Himpens et al. ${ }^{(17)}$ and Weiner et al. (10) showed worsening GERD symptoms early after SG but resolution at 2-3 years. In our study, all symptomatic patients ( 8 patients) were treated with PPIs after diagnosis of GERD. On 6 month follow up symptomatic improvement occurred in $3 / 8(37.5 \%)$ improved. 5 patients $(62.5 \%)$ still not completely improved. Some surgeons considered a hiatal hernia or preexisting GERD to be a contraindication to SG, but we do not necessarily share that sentiment ${ }^{(25)}$.

Physiologic and anatomic effects of SG on GERD were shown in several investigators commented on the anatomic and physiologic effects of SG and postulated their effect on GERD. Hamoui et al. noted the alteration in the anatomy of the angle of His and recommended exercising caution when offering open SG to patients with GERD ${ }^{(26)}$. This is in contrast to the findings of Himpens et al. ${ }^{(17)}$.

Melissas et al. realized an acceleration of gastric emptying in both the short term (6 months) and long term (24 months) after SG. They ventured that surgical division of the ligaments around the abdominal esophagus and destruction of the cardioesophageal junction might account for worsening GERD symptoms (24).

Regarding complications, intraoperative tear of the splenic capsule occurred with bleeding. This was controlled laparoscopically with Argon plasma Coagulation.

Regarding complications directly related to sleeve gasterectomy, there were no leaks, no strictures, no gastrointestinal bleeding, and no perioperative deaths. There were no conversions to an open procedure. A validated questionnaires and objective measurements are recommended at different points to quantify the course of GERD after SG.
Patients with GERD who are possible candidates for SG should be informed of the equivocal evidence regarding the effect of SG on GERD.

A large scale study with large sample size is recommended to further identify the relation between SG and new onset GERD.

\section{CONCLUSION}

In conclusion, there is an agreement with other reports about importance of exclusion of GERD or hiatal hernia preoperatively. We recommend endoscopy before any operation for obesity to exclude hiatal hernia, esophagitis or Barrett esophagus.

Our finding of GERD occurrence was $26.67 \%$ (8/30) of patients. So, if GERD with erosive esophagitis or Barrett esophagus or incompetent LES is diagnosed, SG shouldn't be done. In these clinical situations that are independent of the BMI, Roux-en-Y gastric bypass is indicated.

Only in a very special group of patients with BMI between 30 and 35 and comorbidities, SG plus other antireflux procedure or hiatal hernia repair is accepted.

\section{REFERENCES}

1. Allison DB (2011): Obesity in adulthood and its consequences for life expectancy. Ann. Intern. Med., 138: 24-32.

2. Chang VW and Lauderdale DS (2015): Income disparity in body mass index and obesity in the United States: 1971-2002. Arch. Intern. Med., 165(18):2122-2128.

3. Jian-Fang L, Dan-Dan $L$ and Zhi-Hong L (2014): Comparison of the long-term results of roux-en-y gastric bypass and sleeve gastrectomy for morbid obesity: a systematic review and meta-analysis of randomized and nonrandomized trials. Surg. Laparosc. Endosc. Percutan. Tech., 24(1): 1-11.

4. Tutuian R (2011): Obesity and GERD: pathophysiology and effect of bariatric surgery. Curr. Gastroenterol. Rep., 13(3): 205-212.

5. Vakil N, van Zanten $S V$, Kahrilas $P$ et al. (2006): The montreal definition and classification of gastroesophageal reflux disease: a global evidence-based consensus. Am. J. Gastroenterol., 101:1900-1920.

6. Helmio M, Victorzon $M$ and Ovaska $J$ (2014): comparison of short-term outcome 
of laparoscopic sleeve gastrectomy and gastric bypass in the treatment of morbid obesity: a prospective randomized controlled multicenter sleeve pass study with 6-month follow-up. Scandinavian Journal of Surgery, 3(3): 1-7.

7. Santoro $S$, Lacombe $A$ and de Aquino CGG (2014): Sleeve gastrectomy with anti-reflux procedures. Einstein, 12(3):287294.

8. Anand G and Katz PO (2008): Gastroesophageal reflux disease and obesity. Rev Gastroenterol. Disorders, 8: 233-239.

9. Laffin M, Johnny C, Richdeep SG et al. (2013): Sleeve gastrectomy and gastroesophageal reflux disease. Hindawi Publishing Corporation Journal of Obesity, 16(1):1-6.

10. Weiner RA, Weiner S, Pomhoff I et al. (2007): Laparoscopic sleeve gastrectomy influence of sleeve size and resected gastric volume. Obes. Surg., 17: 1297-1305.

11. Afshin A and Forouzanfar MH (2017): Health effects of overweight and obesity in 195 countries over 25 years. N. Engl. J. Med., 377:13-22.

12. Ogden CL, Carroll MD, Lawman HG et al. (2016): Trends in obesity prevalence among children and adolescents in the United States. JAMA., 315:2292-2300.

13. Viscido G, Gorodner V, Signorini F et al. (2018): Laparoscopic sleeve gastrectomy: endoscopic findings and gastroesophageal reflux symptoms at 18-month follow-up. Journal of Laparoendoscopic and Advanced Surgical Techniques, 28(1):7177.

14. Coupaye M, Gorbatchef C, Calabrese D et al. (2018): Gastroesophageal reflux after sleeve gastrectomy: a prospective mechanistic study. Obesity Surgery, 28(3):838-845.

15. Deitel M, Gagner M, Erickson AL et al. (2011): Third international summit: current status of sleeve gastrectomy. Surg. Obes. Relat. Dis., 7:749-759.

16. Sarela AI, Dexter SP, O'Kane M et al. (2012): Long term follow-up after laparoscopic sleeve gastrectomy: 8-9 - year results. Surg. Obes. Relat. Dis., 8:679-84.

17. Himpens J, Dobbeleir J and Peeters G (2010): Longterm results of laparoscopic sleeve gastrectomy for obesity. Ann Surg., 252:319-234.

18. Santoro $S$ (2007): Technical aspects in sleeve gastrectomy. Obes. Surg., 17:15341539.

19. Gorodner V, Buxhoeveden R, Clemente G et al. (2014): Does laparoscopic sleeve gastrectomy have any influence on gastroesophageal reflux disease? Preliminary results. Surg. Endosc., 29(7): 1760-1768.

20. Rebecchi F, Allaix ME, Giaccone $\mathrm{C}$ et al. (2014): Gastroesophageal reflux disease and laparoscopic sleeve gastrectomy: a physiopathologic evaluation. Ann. Surg., 260:909-923.

21. Braghetto I, Csendes A, Korn O et al. (2010): Gastroesophageal reflux disease after sleeve gastrectomy. Surgical Laparoscopy Endoscopy and Percutaneous Techniques, 20(3): 148-153.

22. Crookes PF (2009): Management of Severe Reflux after Sleeve Gastrectomy. Abstracts of $2^{\text {nd }}$ Annual International Consensus Summit on Sleeve Gastrectomy (ICSSG), Miami Beach (FL, USA).pp: 1921.

23. Nocca D, Krawczykowsky $D$ and Bomans B (2008): A prospective multicenter study of 163 sleeve gastrectomies: results at 1 and 2 years. Obes. Surg., 18:560 -565.

24. Melissas J, Daskalakis $M$ and Koukouraki S (2008): Sleeve gastrectomy a "food limiting" operation. Obes. Surg., 18:1251- 1257.

25. Kakoulidis TP, Karringer A, Gloaguen T et al. (2009): Initial results with sleeve gastrectomy for patients with class I obesity (BMI $30-35 \mathrm{~kg} / \mathrm{m}^{2}$ ). Surg. Obes. Relat. Dis., 5:425-433.

26. Hamoui N, Anthone GJ, Kaufman HS et al. (2006): Sleeve gastrectomy in the highrisk patient. Obes. Surg., 16(11):14451453. 